Monatsschr Kinderheilkd 2012 · 160:215-216 DOI 10.1007/s00112-011-2544-x

Online publiziert: 9 . Februar 2012

(c) Springer-Verlag 2012

\author{
N. Wagner ${ }^{1} \cdot$ F. Zepp ${ }^{2}$ \\ ${ }^{1}$ Klinik für Kinder- und Jugendmedizin, RWTH Aachen, Aachen \\ ${ }^{2}$ Zentrum für Kinder- und Jugendmedizin, Universitätsmedizin, Johannes-Gutenberg-Universität Mainz
}

\title{
Pädiatrische Rheumatologie
}

schrieben wurde, ist selten, aber schwer zu behandeln. Frosch et al. gehen in ihrem Beitrag auf die neuen Aspekte der Pathophysiologie dieser Erkrankung ein. Die SJIA wird heute als Erkrankung des angeborenen Immunsystems im Sinne eines autoinflammatorischen Syndroms aufgefasst. Autoantikörper- oder antigenspezifische T-Zellen als Vertreter des erworbenen Immunsystems lassen sich bei ihr nicht als auslösend finden. Demgegenüber zeigen Betroffene eine deutliche Aktivierung von Zellen des angeborenen Immunsystems. Frosch et al. gehen auf die besondere Bedeutung der verschiedenen Zytokine wie IL-1 (Interleukin 1) und IL-6 sowie die Kalzium bindenden Proteine S100-A8 und deren Bedeutung für die Diagnostik ein. Daran schließen sich die Beschreibung der Klinik der SJIA und schließlich die Darstellung der therapeutischen Möglichkeiten an.

\section{》) Biologicals können das klinische Outcome bei JIA wesentlich verbessern}

In den letzten Jahren wurde nahezu jährlich ein neues Biological für die Therapie rheumatischer Erkrankungen im Kindesalter zugelassen. Klaus Tenbrock stellt die immunologischen Mechanismen vor, die einer Therapie mit Antikörpern oder löslichen Fusionsproteinen gegen IL-1, IL-6 und TNF (Tumornekrosefaktor) zugrunde liegen. Der TNF-Antagonist Etanercept ist sicher das am längsten bekannte und gut untersuchte Medikament dieser Reihe, andere Substanzen wie Adalimumab, Anakinra und Tocilizumab werden von Herrn Tenbrock umfassend in ihrer Wirkweise, Indikation und Verursachung unerwünschter Wirkungen dargestellt, wobei zunächst die für die Therapie der JIA zugelassenen und dann die nichtzugelassenen Substanzen aufgeführt sind. Ein weiteres interessantes Therapiekonzept ist Abatacept, ein Fusionsmolekül aus dem humanen CTLA-4 („cytotoxic t-lymphocyte antigen 4") und einem Anteil des humanen IgG-Rezeptors (IgG: Immunglobulin G). Es verdrängt das kostimulatorische $\mathrm{CD} 28$ aus dessen Bindung an CD80/CD86. Der Autor vertritt die Auffassung, dass mittels der Biologicals eine wesentliche Verbesserung des klinischen Outcomes bei Patienten mit JIA zu erreichen ist.

Der Übergang von chronisch kranken Patienten aus dem jugendlichen in das Erwachsenenalter hat einen deutlichen Einfluss auf die Krankheitsbewältigung. Dieser Prozess wird als Transition bezeichnet. Gerd Ganser et al. stellen in ihrem Beitrag dar, wie wichtig eine interdisziplinäre oder koordinierte Transition für die erfolgreiche Behandlung eines Patienten mit einer JIA ist. Die Autoren werben für ein multidisziplinäres Betreuungskonzept mit dem Ziel einer koordinierten und kontinuierlichen Versorgung von Jugendlichen beim Übergang von der pädiatrischen in die erwachsenenorientierte Betreuung. Sie identifizieren potenzielle Barrieren für eine erfolgreiche Transition sowohl beim Patienten als auch den Angehörigen sowie den Kinder- und internistischen Rheumatologen. Nur eine gemeinsame Anstrengung der verschiedenen Beteiligten an der Transition wird zu einer erfolgreichen Überleitung in die Erwachsenenversorgung chronisch Kranker führen. Die Autoren zeigen auf, dass für solche Transitionsprozesse Ressourcen, beispielsweise in der gemeinsamen Führung von Übergangssprechstunden unter Beteiligung von Pädiatern und Internisten, erforderlich sind. Sie sehen kritisch, dass für diese Prozesse bisher seitens der Kostenträger keine ausreichenden Mittel zur Verfügung stehen. Positiv zu bemerken 
ist, dass es bereits eine erfreulich große Zahl von kinderrheumatologischen Einrichtungen gibt, die über eine Transitionssprechstunde verfügen.

Der Frage zur Prognose der JIA widmen sich Kirsten Minden und Martina Niewerth vom Deutschen Rheumaforschungszentrum Berlin, indem sie auf die Kerndokumentation rheumakranker Kinder und Jugendlicher zurückgreifen. Diese bundesweite Erfassung von über 5000 Kindern mit JIA pro Jahr, die seit mehr als 10 Jahren erfolgt, belegt, dass die Langzeitprognose der JIA heute eindeutig besser als früher ist. Die Patienten erhalten frühzeitiger Basismedikamente, z.B. Methotrexat sowie auch Biologicals, z.B. Etanercept. Dennoch zeigt die Hälfte der Patienten als junger Erwachsener eine aktive Erkrankung, und etwa 30\% der Patienten mit JIA weisen eine Funktionsminderung sowie artikuläre oder extraartikuläre Folgeschäden auf.

Wir hoffen, Ihr Interesse für das Schwerpunktthema Pädiatrische Rheumatologie geweckt zu haben. Die Patienten mit rheumatischen Erkrankungen profitierten in den letzten Jahren eindeutig davon, dass sich zunehmend mehr Kinderärzte mit dieser Thematik intensiv vertraut machen und daher durch eine frühere Diagnosestellung und verbesserte Behandlungsmöglichkeiten die Prognose der Patienten optimiert werden konnte.

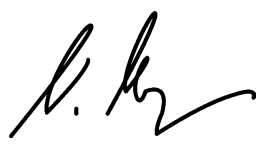

Prof. Dr. N. Wagner

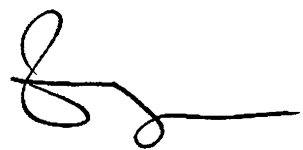

Prof. Dr. F. Zepp

\section{Korrespondenzadresse}

\section{Prof. Dr. N. Wagner}

Klinik für Kinder- und Jugendmedizin, RWTH Aachen

Pauwelsstraße 30, 52074 Aachen

nwagner@ukaachen.de
Maier R.F., Obladen M. (Hrsg) Neugeborenenintensivmedizin Evidenz und Erfahrung

Berlin Heidelberg New York: Springer 2011, 8. Auflage, 613 S., 63 Abb., 89 Tab., (ISBN 978-3-642-01068-2), 29.95 EUR

$\begin{array}{ll} & \begin{array}{l}\text { Fünf Jahre nach der } \\ \text { letzten Auflage ist } \\ \text { Hier steht eine Anzeige. } \\ \text { also nun Der Ob- } \\ \text { laden in neuer und } 8 .\end{array} \\ & \text { Auflage erschienen. } \\ & \text { Schon auf den ersten } \\ & \text { Blick fällt auf, dass die } \\ & \text { Herausgeber ihre Rei- } \\ & \text { henfolge getauscht }\end{array}$

haben und nun Rolf F. Maier an erster Stelle erscheint, während sich Michael Obladen an die zweite Stelle zurückgezogen hat.

Das Kitteltaschenformat wurde unverändert beibehalten, wobei allerdings die neue Ausgabe deutlich schlanker ist als die Vorausgabe aus dem Jahr 2006. Damit eignet sich das Buch wirklich wieder für die Kitteltasche. Gelungen ist dies einerseits, indem einzelne selbstverständlich gewordene Inhalte weggelassen wurden und dadurch eine Seitenreduktion von 678 auf jetzt 613 Seiten erreicht wurde, andererseits durch eine Änderung der Papierqualität bzw. -stärke.

Auch inhaltlich wurde der Aufbau etwas umstrukturiert, und die Kapitelzahl wurde von 22 auf 18 reduziert. Insbesondere im allgemeinen Teil sind die Kapitelbezeichnungen und deren Abfolge geändert, während der Organteil größtenteils belassen wurde. An die Stelle von „Aufbau und Organisation einer Neugeborenenintensivstation" trat das durchaus zeitgemäße Thema „Regionalisierung, Qualitätssicherung, Ergebnisse".

In ihrem Vorwort betonen die Herausgeber, dass es für viele Gebiete der Neonatologie keine gesicherte Evidenz gibt und dass es sich daher bei den Therapiekonzepten in ihrem Buch vielfach nur um individuelle und auf klinischer Erfahrung beruhende (und damit unverbindliche) Empfehlungen handelt. Sichtbar wird das z. B. bei der Empfehlung von Chloramphenicol als Zweitlinientherapie bei Neugeborenensepsis, einem Medikament, das wegen seines Nebenwirkungspotenzials z. B. in Österreich für systemische Anwendungen gar nicht mehr verfügbar ist. In seiner Konzeption ist das Buch übersichtlich, und auch der noch nicht Eingelesene findet rasch die aktuell benötigten Hinweise.
Dabei ist der Text insgesamt knapp gehalten, sodass man sich die gesuchten Informationen in kurzer Zeit aneignen kann. Die 63 Abbildungen und 89 Tabellen sind übersichtlich gestaltet und ergänzen den Text in idealer Weise.

Das Buch kann durch seine komprimierte Darstellungsweise naturgemäß Standardwerke der Neonatologie wie jene von Polin, Rennie, Volpe usw. nicht ersetzen, und die Autoren selbst weisen durch eine Listung weiterführender Literatur im Einleitungsteil auch dezidiert hierauf hin.

Bei der insgesamt sehr knappen Darstellungsweise stellt sich die Frage, ob es wirklich notwendig ist, jedem Kapitel eine umfangreiche Literaturliste (bis zu 10 Seiten!) folgen zu lassen. Diesbezüglich wäre evtl. eine Leserbefragung über den Nutzen dieser Referenzen im klinischen Alltag sinnvoll. Eine Auslagerung der Literaturzitate in eine webbasierte Datenbank (evtl. mit Verlinkung zu den Abstracts bzw. zu PubMed) ist wohl eine Überlegung wert. - Dadurch würde das Buch noch besser in die Kitteltasche passen ....

Es wird wohl ein wenig Zeit brauchen, bis Der Maier im Alltagssprachgebrauch die Stelle Des Obladen eingenommen haben wird. Bleiben wird die Tatsache, dass dieses Buch im deutschsprachigen Raum ein Standardwerk für neonatologisch Tätige darstellt. Da an neonatologischen Abteilungen wohl immer die neueste Version des Buchs aufliegen sollte, wird auch diese Auflage ausreichend Abnehmer finden. Die im Internetbuchhandel zu bezahlenden 29,95 EUR sind jedenfalls sinnvoll investiert.

Reinhold Kerbl (Leoben) 